\title{
Double heavy tri-hadron bound state with strange flavor
}

\author{
Li $\mathrm{Ma}^{1, *}$ \\ ${ }^{1}$ Helmholtz-Institut für Strahlen- und Kernphysik and Bethe Center for Theoretical Physics, Universität \\ Bonn, D-53115 Bonn, Germany
}

\begin{abstract}
Through the Born-Oppenheimer Approximation, we have performed a comprehensive investigation of the $D D^{*} K, D \bar{D}^{*} K, B B^{*} \bar{K}$ and $B \bar{B}^{*} \bar{K}$ molecular states. In the framework of One-Pion Exchange model as well as the treatments of the coupled-channel effects and S-D wave mixing, we find a loosely bound tri-meson molecular state these systems with the isospin configuration $\left|0, \frac{1}{2}, \pm \frac{1}{2}\right\rangle$ and quantum number $I\left(J^{P}\right)=1 / 2\left(1^{-}\right)$, where the $\frac{1}{2}$ is the total isospin of the three-body system, the 0 is the isospin of the $D^{*} K, \bar{D}^{*} K, B^{*} \bar{K}$ and $\bar{B}^{*} \bar{K}$. With the estimated error, the mass of the $D D^{*} K$ or $D \bar{D}^{*} K$ molecule is $4317.92_{-4.32}^{+3.66} \mathrm{MeV}$ or $4317.92_{-6.55}^{+6.13} \mathrm{MeV}$. We also extend our calculations to the bottom sector and find tri-meson bound states for the $B B^{*} \bar{K}$ and $B \bar{B}^{*} \bar{K}$ with the mass $11013.65_{-8.84}^{+8.49} \mathrm{MeV}$ and $11013.65_{-9.02}^{+8.68} \mathrm{MeV}$ respectively.
\end{abstract}

\section{Introduction}

The investigations on the three body systems have been an important issue in recent years. The explorations on the few-body systems were first proposed to focus on the few nucleon systems or nucleons and hyperons [1-4]. Systematical calculations on the resonances containing three mesons were also studied [5-7]. The $X(2175)$ as a resonant state of the $\phi K \bar{K}$ system was stated in [5]. Theoretical study on the $K K \bar{K}$ system and dynamical generation of the $K(1460)$ resonance was performed in [6]. Theoretical support for the $\pi(1300)$ and the reported $f_{0}(1790)$ as a candidate of $f_{0}(980) \pi \pi$ molecular resonances were claimed in [7]. Theoretical interpretations on some low lying excited baryons could be reproduced by the resonances composed of two mesons and one baryon were also be proposed in [8-10]. Many theorists have extended the investigations on the three body systems to the charm sector. The description of the $Y(4260)$ as a resonant state of $J / \psi K \bar{K}$ system stated in [11]. The energy and width of a narrow $I=1 / 2 D N N$ quasibound state had been discussed in [12]. The studies on the $N D K, \bar{K} D N$ and $N D \bar{D}$ molecules publicised in [13]. The $N \bar{K} K$ system and the signature of a $N^{*}(1920)\left(1 / 2^{+}\right)$state were studied and the Fixed Center Approximation to the Faddeev equations was used in [14]. A complete description of the $D K K$ and $D K \bar{K}$ with the Fixed Center Approximation was also discussed in [15]. The systems were also studied in [16, 17]. Very recently, the theoretical calculations on the $B D \bar{D}$ and $B D D$ systems were performed, and the hints of a bound state in the energy region $8935-8985 \mathrm{MeV}$ with the quantum numbers $I\left(J^{P}\right)=1 / 2\left(0^{-}\right)$for the $B D \bar{D}$ system was claimed [18]. E. Wilbring et al. had calculated the molecule-meson scattering amplitudes for the $Z_{b}(10610)$ or $Z_{b}(10650)$ by employing the molecule assumptions and the dimmer tricks [19]. They had predicted the phase shifts for

\footnotetext{
*e-mail: ma@hiskp.uni-bonn.de
} 
the scattering of $B$ and $B^{*}$ mesons off the exotic mesons $Z_{b}(10610)$ and $Z_{b}(10650)$, and the possibility for universal bound states of three $B$ and $B^{*}$ mesons form the Efimov effect had been ruled out. Moreover, the three-particle Efimov states in a finite volume via the dimmer formalism had been extensively explored in [20,21].

The one pion exchange model (OPE) has been widely used to study the di-meson or dibaryon molecular systems. Since the pion has the lowest mass, longest effect range and the constituent hadrons should be well separated, the long range one-pion-exchange should play a dominant role among the possible exchange mesons. It is reasonable to extend the OPE model to a system containing three mesons, if we only focus on searching for its loosely bound states. The Born-Oppenheimer Approximation has been employed to study the fewbody systems containing several heavy particles and several light particles [22]. For a threeparticle system, it allows us to separate the system into two part. One part is the motion of the light particles relative to the heavy particles with fixed locations; The other part is the relative motion between the heavy particles. The Born-Oppenheimer Approximation has also been used to explore the properties of the di-meson molecules [23].

The di-meson and di-baryon molecules are formed by the effective potential produced by the pion exchange. To a very good extent, it is a pion shared by the two constitutes providing an attraction which makes them bound. It can be regarded as a $\sigma$ bond similar to the $\sigma$ bond in hydrogen molecules. There is another kind of bond called $\pi$ bond universally existing in benzene molecules, which is a pair of electrons shared by the six carbon atoms. Therefore, it is fascinating that whether there exists a hadronic $\pi$ bond in a system containing more than two hadrons. Specifically, whether there exist hadronic $\pi$ bond in the tri-meson systems like $D D^{*} K, D \bar{D}^{*} K, B B^{*} \bar{K}$ and $B \bar{B}^{*} \bar{K}$.

\section{One-pion-exchange model and the two-body sector}

The OPE interaction means there should be only one pion exchanged by any two constituents. The $D D^{*} K$ or $D \bar{D}^{*} K$ sharing one virtual pion seems like a benzene molecule with an electron pair shared by its six carbon atoms. We call the interaction produced by one pion shared by more than two hadrons as a hadronic $\pi$ bond. However, the conservations of the angular momentum and C parity don't allow the direct channel of the $D K, D^{*} K$ and $\bar{D}^{*} K$. Thus, the interactions in the $D D^{*} K$ or $D \bar{D}^{*} K$ system are dominant by the coupled-channel effects. Specifically, if we use $a, b$ and $c$ to label the locations of the three distinct mesons in the original channel, which is $D_{a} D_{b}^{*} K_{c}\left(D_{a} \bar{D}_{b}^{*} K_{c}\right)$. It can change into $D_{a} D_{b} K_{c}^{*}\left(D_{a} \bar{D}_{b} K_{c}^{*}\right)$ via one-pion-exchange between $b$ and $c$. While the channel $D_{a} D_{b} K_{c}^{*}\left(D_{a} \bar{D}_{b} K_{c}^{*}\right)$ can also change into $D_{a}^{*} D_{b} K_{c}\left(D_{a}^{*} \bar{D}_{b} K_{c}\right)$ through a pion exchanged between $a$ and $c$. When a pion arises between $a$ and $b$, the channel $D_{a}^{*} D_{b} K_{c}\left(D_{a}^{*} \bar{D}_{b} K_{c}\right)$ returns back into the original channel $D_{a} D_{b}^{*} K_{c}$ $\left(D_{a} \bar{D}_{b}^{*} K_{c}\right)$. Within this scenario, one might expect that the three channels are coupled together to form a loosely bound state. The same analysis can also be extended to the $B B^{*} \bar{K}$ and $B \bar{B}^{*} \bar{K}$ systems.

The Lagrangians with the SU(2) chiral symmetry and C parity conservation read

$$
\mathcal{L}_{P}=-i \frac{2 g}{f_{\pi}} \bar{M} P_{b}^{* \mu} \partial_{\mu} \phi_{b a} P_{a}^{\dagger}+i \frac{2 g}{f_{\pi}} \bar{M} P_{b} \partial_{\mu} \phi_{b a} P_{a}^{* \mu^{\dagger}}
$$

where the heavy flavor meson fields $P^{(*)}$ represent $P^{(*)}=\left(D^{(*) 0}, D^{(*)}\right),\left(B^{(*)-}, \bar{B}^{(*) 0}\right)$ or $\left(K^{(*)-}, \bar{K}^{(*) 0}\right)$. The $\phi$ in the above represents the exchanged pion matrix:

$$
\phi=\left(\begin{array}{cc}
\frac{1}{\sqrt{2}} \pi^{0} & \pi^{+} \\
\pi^{-} & -\frac{1}{\sqrt{2}} \pi^{0}
\end{array}\right) .
$$


In the OPE model, there are three coupling coefficients need to be determined. One is the pion decay constant $f_{\pi}=132 \mathrm{MeV}$, we got from chiral symmetry. And one is the pionic coupling constant $g=0.59$ extracted from the width of $D^{*+}[24]$. The other one is the coupling constant $g^{\prime}$. Here, we take the experimental result $g^{\prime}=0.90$ which was obtained from the full widths of $K^{*}$ in the PDG [24].

In the calculations, each vertex in the Feynman diagram needs a form factor to suppress the high momentum contribution. We take the conventional form for the form factor as in the Bonn potential model, which is $F(q)=\frac{\Lambda^{2}-m_{\pi}^{2}}{\Lambda^{2}-q^{2}}=\frac{\Lambda^{2}-m_{\pi}^{2}}{\tilde{\Lambda}^{2}+\vec{q}^{2}}$, where $m_{\pi}$ is the mass of the exchanged meson and $\tilde{\Lambda}^{2}=\Lambda^{2}-\left(\Delta M^{(\prime)}\right)^{2}, \Delta M=\left\{\left(M_{D}^{* 2}+M_{K}^{* 2}\right)-\left(M_{D}^{2}+M_{K}^{2}\right)\right\} /\left\{2\left(M_{D}+M_{K}^{*}\right)\right\}$ and $\Delta M^{\prime}=\left\{\left(M_{B}^{* 2}+M_{K}^{* 2}\right)-\left(M_{B}^{2}+M_{K}^{2}\right)\right\} /\left\{2\left(M_{B}+M_{K}^{*}\right)\right\}$. The role of the form factor is to remove or suppress the contribution from the ultraviolet region of the exchanged momentum.

After carefully solving the couple-channel Schrödinger equation with the treatment of the S-D wave mixing, we find that for the $D^{*} K$ system, the isospin $I=1$ case gives a repulsive potential, obviously, it has no bound solution. While the isospin $I=0$ case corresponds to an attractive potential then has a bound state. For the $B^{*} \bar{K}$ system, we find out a loosely bound state for the $B^{*} \bar{K}$ with its isospin $I=0$. We assume the $D_{s 1}(2460)$ reported in the experiment is dominant by a loosely bound $D^{*} K$ molecule [25]. The cutoff parameter could be fixed definitely at $803.20 \mathrm{MeV}$. Then the binding energy and the root-mean-square radius of the bound state obtained are $42.29 \mathrm{MeV}$ and $1.14 \mathrm{fm}$, respectively. The dominant component is the $\mathrm{S}$ wave with a probability $98.92 \%$. While the corresponding component from $\mathrm{D}$ wave is $1.08 \%$. Numerically, the probability of the component $D^{*} K$ is $75.83 \%$ while that of the component $D K^{*}$ is $24.17 \%$. We also adopt the speculations proposed in [26], that the $D_{s 1}(2460)$ has a counter partner state in bottom meson sector. It might be a good candidate of the $B^{*} \bar{K}$ molecular state with mass $5778 \mathrm{MeV}$. Thus its binding energy might be $42.19 \mathrm{MeV}$. Similar to the treatment for the $D^{*} K$, we fix the cutoff parameter at $1451.00 \mathrm{MeV}$. Then the binding energy and the root-mean-square radius of the bound state obtained are $42.19 \mathrm{MeV}$ and $0.96 \mathrm{fm}$, respectively. The probability for the $\mathrm{S}$ wave and D wave component are $99.32 \%$ and $0.68 \%$. The probability of the component $B^{*} \bar{K}$ is $78.66 \%$ while that of the component $B \bar{K}^{*}$ is $21.34 \%$.

\section{Three-body sector and Born-Oppenheimer Approximation}

Based on the main idea of the BOA, there are two kinds of degree of freedoms in the $D D^{*} K$ system as an example, the motion of the $K$ related to the $D$ and $D^{*}$, and the relative motion between $D$ and $D^{*}$. In the Born-Oppenheimer Approximation, both of the two degrees of freedom are independent, and they can be treated separately. Therefore, the total wave functions of the three-body system $D D^{*} K$ can be expressed as

$$
\begin{aligned}
\left|\Psi_{\text {Total }}\right\rangle & =C_{0}\left\{\varphi(\vec{R}) \psi\left(\overrightarrow{r_{b}}\right)\left|D_{a} D_{b}^{*} K_{c}\right\rangle+C \varphi^{\prime}(\vec{R})\left[\psi^{\prime}\left(\overrightarrow{r_{a}}\right)+\psi^{\prime}\left(\overrightarrow{r_{b}}\right)\right]\left|D_{a} D_{b} K_{c}^{*}\right\rangle\right. \\
& \left.+\varphi(\vec{R}) \psi\left(\overrightarrow{r_{a}}\right)\left|D_{a}^{*} D_{b} K_{c}\right\rangle\right\}
\end{aligned}
$$

where the $\varphi(\vec{R})$ and $\varphi^{\prime}(\vec{R})$ are normalized, which satisfy $\int \varphi^{*}(\vec{R}) \varphi(\vec{R}) d \vec{R}=1$, $\int \varphi^{\prime *}(\vec{R}) \varphi^{\prime}(\vec{R}) d \vec{R}=1$. The $C_{0}$ is the normalization coefficient. The wave functions $\psi\left(\overrightarrow{r_{a}}\right)$ and $\psi^{\prime}\left(\overrightarrow{r_{a}}\right)$ can be determined in the last section. The $C$ is a variation parameter.

The total Hamiltonian of the three-body system $D D^{*} K$ is

$$
H_{T}=T_{h}+V_{h}+T_{K}+V_{K},
$$


where the $T_{h}$ and $V_{h}$ denote the relative kinetic energy and the effective potential between the $D$ and $D^{*}$ respectively. The $T_{K}$ and $V_{K}$ is the kinetic energy and effective potential of the $K$ in the $D D^{*} K$ system. The Schrödinger equation for the three-body system can be simplified as

$$
E_{3}|\Phi(\vec{R})\rangle=H_{T}^{\prime}|\Phi(\vec{R})\rangle=\left[T_{h}(\vec{R})+V_{h}(\vec{R})+V_{B O}(\vec{R})\right]|\Phi(\vec{R})\rangle
$$

where $H_{T}^{\prime}=H_{T}-E_{b}$. The reduced energy $E_{3}=E_{T}-E_{b}$.

After solving the Schrödinger equation for the three-body system, we find out the loosely bound states for the $D D^{*} K$ and $D \bar{D}^{*} K$ with the isospin configuration $\left|0, \frac{1}{2}, \pm \frac{1}{2}\right\rangle$. For the system $D D^{*} K$ and $D \bar{D}^{*} K$, if we assume the $D_{s 1}(2460)$ is molecular state of $D^{*} K$ respectively, then the cutoff parameters $\Lambda$ can be fixed at $803.20 \mathrm{MeV}$. The reduced three-body binding energy $\left|E_{3}\right|$ for the $D D^{*} K\left(D \bar{D}^{*} K\right)$ is $8.29 \mathrm{MeV}$, and the root-mean-square radius for the $D$ and $D^{*}\left(\bar{D}^{*}\right)$ is $1.65 \mathrm{fm}$. For the $D D^{*} K$, the isospin configuration $\left|0, \frac{1}{2}, \frac{1}{2}\right\rangle$ contains the $D^{+} D^{*+}$ and $D^{+} D^{* 0}$, the $\left|0, \frac{1}{2},-\frac{1}{2}\right\rangle$ contains the $D^{0} D^{*+}$ and $D^{0} D^{* 0}$. Taking into account the effective potentials and the channel-dependent coefficients, we find the effective potentials for the $D D^{*}$ are cancelled out. Thus, only the BO potential provided by the $K$ effect the three-body system. For the $D \bar{D}^{*} K$, the isospin configuration $\left|0, \frac{1}{2}, \frac{1}{2}\right\rangle$ contains the $D^{+} \bar{D}^{* 0}$ and $D^{+} D^{*-}$, the $\left|0, \frac{1}{2},-\frac{1}{2}\right\rangle$ contains the $D^{0} \bar{D}^{* 0}$ and $D^{0} D^{*-}$. Similarily, without the BO potential, the four channel have no possibility to be bound states.

The $B B^{*} \bar{K}$ and $B \bar{B}^{*} \bar{K}$ also have a loosely bound states with the isospin configuration $\left|0, \frac{1}{2}, \pm \frac{1}{2}\right\rangle$. For the system $B B^{*} \bar{K}$ and $B \bar{B}^{*} \bar{K}$, if we assume there exists a $B^{*} \bar{K}$ molecule at 5778 $\mathrm{MeV}$ as a counter-partner of the $D^{*} K$, then the cutoff parameter $\Lambda^{\prime}$ can be fixed at 1451.00 $\mathrm{MeV}$. The reduced three-body binding energy $\left|E_{3}\right|$ for the $B B^{*} \bar{K}\left(B \bar{B}^{*} \bar{K}\right)$ is $41.76 \mathrm{MeV}$, and the root-mean-square radius for the $B$ and $B^{*}\left(\bar{B}^{*}\right)$ is $0.65 \mathrm{fm}$. For the $B B^{*} \bar{K}$, the isospin configuration $\left|0, \frac{1}{2}, \frac{1}{2}\right\rangle$ contains the $B^{+} B^{*+}$ and $B^{+} B^{* 0}$, the $\left|0, \frac{1}{2},-\frac{1}{2}\right\rangle$ contains the $B^{0} B^{*+}$ and $B^{0} B^{* 0}$. For the $B \bar{B}^{*} \bar{K}$, the isospin configuration $\left|0, \frac{1}{2}, \frac{1}{2}\right\rangle$ contains the $B^{+} \bar{B}^{* 0}$ and $B^{+} B^{*-}$, the $\left|0, \frac{1}{2},-\frac{1}{2}\right\rangle$ contains the $B^{0} \bar{B}^{* 0}$ and $B^{0} B^{*-}$. Similar with the charm systems, the BO potential plays an important role to make them bound.

The discussion on the $D D^{*} K$ and $D \bar{D}^{*} K$ molecules above are based on the assumption that the reported state $D_{s 1}(2460)$ can fit into the $D^{*} K$ molecule scenario, then the cutoff parameter $\Lambda$ can be fixed at $803.20 \mathrm{MeV}$. It is interesting to investigate the sensitivity of the three-body eigen-energy to the variance of the cutoff parameter $\Lambda$. The numerical results on the $B B^{*} \bar{K}$ and $B \bar{B}^{*} \bar{K}$ molecules above are based on the hypothesis that there exists a $B^{*} \bar{K}$ molecule at $5778 \mathrm{MeV}$ as a counter-partner of the $D^{*} K$, then the cutoff parameters $\Lambda^{\prime}$ can be fixed at $1451.00 \mathrm{MeV}$. We plot dependence of the three body binding energy $\left|E_{3}\right|$ on the two body binding energy $\left|E_{b}\right|$ for the $D D^{*} K$ and $D \bar{D}^{*} K$ in Figure 1 . That dependence for the $B B^{*} \bar{K}$ and $B \bar{B}^{*} \bar{K}$ also plotted in Figure 1. Where the red and green bands are from PDG2016 and PLB647,133, respectively. The three-body binding energies are $E_{I=1 / 2}^{D D^{*} K}=8.29_{-3.66}^{+4.32} \mathrm{MeV}$, $E_{I=1 / 2}^{B B^{*} \bar{K}}=41.76_{-8.84}^{+8.49} \mathrm{MeV}$. The error band is estimated as $m_{K} /\left(2 m_{u}\right)$ with $m_{u}$ the reduced mass of the two heavy particles. As the long-distance $D D^{*}$ potential is related to that of the $D \bar{D}^{*}$ potential by $G$-parity [27], there could also exist a three-body $D \bar{D}^{*} K$ bound state, but with additional uncertainty $m_{\pi}^{2} /\left(2 m_{u}\right)$ (which characterizes the natural energy scale of OPE [28]) from the unknown short-distance interaction. Thus, for the $D \bar{D}^{*} K$ and $B \bar{B}^{*} \bar{K}$ system, the three-body binding energies are $E_{I=1 / 2}^{D \bar{D}^{*} K}=8.29_{-6.13}^{+6.55} \mathrm{MeV}, E_{I=1 / 2}^{B \bar{B}^{*} \bar{K}}=41.76_{-9.02}^{+8.68} \mathrm{MeV}$. With the additional uncertainty from the missing short-distance interaction. Those correspond to two bound states with masses $4317.92_{-6.55}^{+6.13} \mathrm{MeV}$ and $11013.65_{-9.02}^{+8.68} \mathrm{MeV}$. 

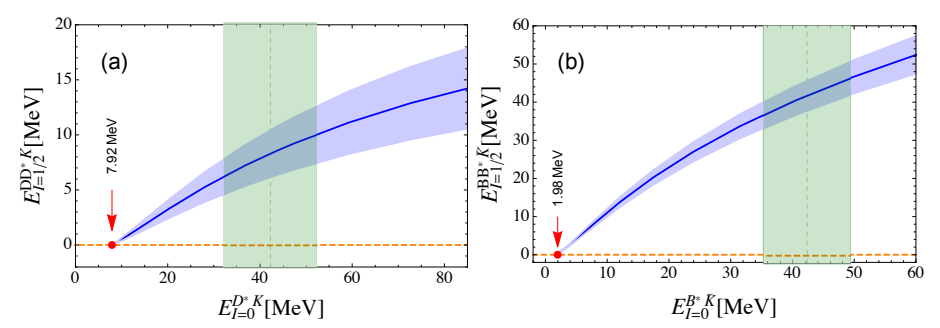

Figure 1. The dependence of the three-body binding energy $\left|E_{3}\right|$ on the two-body binding energy $\left|E_{b}\right|$. (a) is for the charm sector. (b) is for the bottom sector.

\section{Summary and Discussion}

In the present paper, we have performed an extensive study on the possibility of the $D D^{*} K$, $D \bar{D}^{*} K, B B^{*} \bar{K}$ and $B \bar{B}^{*} \bar{K}$ in forming the tri-meson molecules. With the hypothesis of the $D_{s 1}(2460)$ as the $D^{*} K$ molecule, the cutoff parameter $\Lambda$ can be fixed at $803.20 \mathrm{MeV}$. After the calculation of the Schrödinger Equation within the Born-Oppenheimer Approximation as well as the OPE scheme, we find out a loosely bound tri-meson molecular state for the $D D^{*} K$ and $D \bar{D}^{*} K$ system with the isospin configuration $\left|0, \frac{1}{2}, \pm \frac{1}{2}\right\rangle$. The reduced three-body binding energy of the $D D^{*} K$ and $D \bar{D}^{*} K$ molecule are $8.29_{-3.66}^{+4.32} \mathrm{MeV}$ and $8.29_{-6.13}^{+6.55} \mathrm{MeV}$. The root-mean-square between $D$ and $K$ as well as $D^{*}\left(\bar{D}^{*}\right)$ and $K$ are both $1.14 \mathrm{fm}$. The rootmean-square between $D$ and $D^{*}\left(\bar{D}^{*}\right)$ is $1.65 \mathrm{fm}$. The root-mean-square structure for the $D D^{*} K$ and $D \bar{D}^{*} K$ seem like a triangle. As we have derived in Sect. 2, the two-body binding energy of the $D^{*} K$ or $\bar{D}^{*} K$ is $42.29 \mathrm{MeV}$. Therefore, the total binding energy of the $D D^{*} K$ and $D \bar{D}^{*} K$ molecules relative to the $D D^{*} K$ threshold are both the $\left|E_{T}\right|=\left|E_{3}+E_{b}\right|$ and equal to $50.58 \mathrm{MeV}$. With the estimated error, the mass of the $D D^{*} K$ and $D \bar{D}^{*} K$ molecules are 4317.92 $2_{-4.32}^{+3.66} \mathrm{MeV}$ and 4317.92 $2_{-6.55}^{+6.13} \mathrm{MeV}$.

Our calculations have indicated that the heavy mesons in the three-body system can't be bound without the BO potential provided by the $K$, since the effective potential of some channels like $D^{+} D^{*+}\left(D^{+} \bar{D}^{* 0}\right)$ and $D^{0} D^{* 0}\left(D^{0} D^{*-}\right)$ are all repulsive. The virtual pion exchanged in the three body system plays a vital role in forming the tri-meson molecules. Its status seems like the $\pi$ bond in the Benzene molecule where the six carbons share a pair of electrons. Obviously, the delocalized $\pi$ bond also exists in the tri-meson molecular states.

We also extend our investigation to the bottom sector. With the assumption that there exists a $B^{*} \bar{K}$ molecule at $5778 \mathrm{MeV}$ as a counter-partner of the $D^{*} K$, then the cutoff parameter $\Lambda^{\prime}$ can be fixed at $1451.00 \mathrm{MeV}$. We also find out a loosely bound tri-meson molecular state for the $B B^{*} \bar{K}$ and $B \bar{B}^{*} \bar{K}$ with the isospin configuration $\left|0, \frac{1}{2}, \pm \frac{1}{2}\right\rangle$. The reduced three-body binding energy $\left|E_{3}\right|$ for the $B B^{*} \bar{K}\left(B \bar{B}^{*} \bar{K}\right)$ is $41.76_{-8.84}^{+8.49} \mathrm{MeV}$. The two-body binding energy of the $B^{*} \bar{K}$ or $\bar{B}^{*} \bar{K}$ is $42.19 \mathrm{MeV}$. Thus, the total binding energy of the $B B^{*} \bar{K}$ and $B \bar{B}^{*} \bar{K}$ molecules relative to the $B B^{*} K$ threshold is the $\left|E_{T}\right|=\left|E_{3}+E_{b}\right|$ and equal to $83.95 \mathrm{MeV}$. With the estimated error, the mass of the $B B^{*} \bar{K}$ and $B \bar{B}^{*} \bar{K}$ molecule are $11013.65_{-8.84}^{+8.49} \mathrm{MeV}$. The root-mean-square radius for the $B$ and $B^{*}\left(\bar{B}^{*}\right)$ is $0.96 \mathrm{fm}$. The root-mean-square radius for the $\bar{K}$ and $B^{*}\left(\bar{B}^{*}\right)$ is $0.65 \mathrm{fm}$. Thus, its root-mean-square structure for the $B B^{*} \bar{K}\left(B \bar{B}^{*} \bar{K}\right)$ also seems like a triangle.

The $D \bar{D}^{*} K$ molecule may be easily detected in experiment. As the experiments on the $B$ meson decay have been well developed. It will provide us a good platform to probe the trimeson molecule $D \bar{D}^{*} K$. The $D \bar{D}^{*} K$ molecule with the isospin $\left|0, \frac{1}{2}, \frac{1}{2}\right\rangle$ and $I\left(J^{P}\right)=1 / 2\left(1^{-}\right)$ has one unit positive charge. It is possible to be probed in the process $B^{+} \rightarrow T(4318)^{+} \rightarrow$ 
$\left(J / \psi \pi^{0} K^{+}\right) \pi^{0}$, or to be detected in the process $B^{0} \rightarrow T(4318)^{+} \rightarrow\left(J / \psi \pi^{0} K^{+}\right) \pi^{-}$. Here we use $T(4318)^{+}$denote the charge partner of the $D \bar{D}^{*} K$ molecular state. The $D \bar{D}^{*} K$ molecule with the isospin $\left|0, \frac{1}{2},-\frac{1}{2}\right\rangle$ is neutral. Its main decay process could be $B^{ \pm} \rightarrow T(4318)^{0} \rightarrow$ $\left(J / \psi \pi^{0} K^{+}\right) \pi^{ \pm}$. The radiative decays $B^{+} \rightarrow T(4318)^{+} \rightarrow\left(J / \psi \pi^{0} K^{+}\right) \gamma$ and $B^{ \pm} \rightarrow T(4318)^{0} \rightarrow$ $\left(J / \psi \pi^{0} K^{0}\right) \gamma$ might be another way to detect the tri-meson molecules. The LHCb, BABAR, Belle, BESIII and other collaborations have accumulated many decay data on the process $B^{ \pm} \rightarrow\left(J / \psi \pi^{+} \pi^{-}\right) K^{ \pm}$. These data mainly analyzed around $3872 \mathrm{MeV}$ in the $J / \psi \pi^{+} \pi^{-}$invariant mass. If we analyze these data with a higher mass at $4312 \mathrm{MeV}$ in the $J / \psi \pi K$ invariant mass, It will unveil the information on the tri-meson system $D \bar{D}^{*} K$. Moreover, the $D D^{*} K$ molecule has possibility to be probed in the forthcoming experiments on the $p p$ collision.

\section{References}

[1] E. O. Alt, P. Grassberger and W. Sandhas, Nucl. Phys. B 2, 167 (1967)

[2] E. Epelbaum, A. Nogga, W. Gloeckle, H. Kamada, U. G. Meissner and H. Witala, Phys. Rev. C 66, 064001 (2002)

[3] A. Deltuva and A. C. Fonseca, Phys. Rev. C 75, 014005 (2007)

[4] E. Hiyama, Y. Kino and M. Kamimura, Prog. Part. Nucl. Phys. 51, 223 (2003).

[5] A. Martinez Torres, K. P. Khemchandani, L. S. Geng, M. Napsuciale and E. Oset, Phys. Rev. D 78, 074031 (2008)

[6] A. Martinez Torres, D. Jido and Y. Kanada-En'yo, Phys. Rev. C 83, 065205 (2011)

[7] A. Martinez Torres, K. P. Khemchandani, D. Jido and A. Hosaka, Phys. Rev. D 84, 074027 (2011)

[8] A. Martinez Torres, K. P. Khemchandani and E. Oset, Phys. Rev. C 77, 042203 (2008)

[9] Y. Kanada-En'yo and D. Jido, Phys. Rev. C 78, 025212 (2008)

[10] A. Martinez Torres and D. Jido, Phys. Rev. C 82, 038202 (2010)

[11] A. Martinez Torres, K. P. Khemchandani, D. Gamermann and E. Oset, Phys. Rev. D 80, 094012 (2009)

[12] M. Bayar, C. W. Xiao, T. Hyodo, A. Dote, M. Oka and E. Oset, Phys. Rev. C 86, 044004 (2012)

[13] C. W. Xiao, M. Bayar and E. Oset, Phys. Rev. D 84, 034037 (2011)

[14] J. J. Xie, A. Martinez Torres and E. Oset, Phys. Rev. C 83, 065207 (2011)

[15] V. R. Debastiani, J. M. Dias and E. Oset, Phys. Rev. D 96, no. 1, 016014 (2017)

[16] D. Jido and Y. Kanada-En'yo, Phys. Rev. C 78, 035203 (2008)

[17] A. Martinez Torres, K. P. Khemchandani and E. Oset, Phys. Rev. C 79, 065207 (2009)

[18] J. M. Dias, V. R. Debastiani, L. Roca, S. Sakai and E. Oset, arXiv:1709.01372 [hep-ph].

[19] E. Wilbring, H.-W. Hammer and U. G. Meißner, arXiv:1705.06176 [hep-ph].

[20] H. W. Hammer, J. Y. Pang and A. Rusetsky, JHEP 1709, 109 (2017)

[21] H.-W. Hammer, J.-Y. Pang and A. Rusetsky, arXiv:1707.02176 [hep-lat].

[22] S. Moroz and Y. Nishida, Phys. Rev. A 90, no. 6, 063631 (2014)

[23] E. Braaten, C. Langmack and D. H. Smith, Phys. Rev. D 90, no. 1, 014044 (2014)

[24] C. Patrignani et al. [Particle Data Group], Chin. Phys. C 40, no. 10, 100001 (2016)

[25] A. Martínez Torres, E. Oset, S. Prelovsek and A. Ramos, JHEP 1505, 153 (2015)

[26] F. K. Guo, P. N. Shen and H. C. Chiang, Phys. Lett. B 647, 133 (2007)

[27] E. Klempt, F. Bradamante, A. Martin and J. M. Richard, Phys. Rept. 368, 119 (2002).

[28] E. Braaten and M. Lu, Phys. Rev. D 76, 094028 (2007) 